\title{
The existence of nontrivial solution of a class of Schrödinger-Bopp-Podolsky system with critical growth
}

Jie Yang ${ }^{1,2}$, Haibo Chen ${ }^{1 *}$ and Senli Liu ${ }^{1}$

"Correspondence:

math_chb@163.com

'School of Mathematics and

Statistics, Central South University,

South Lushan Road, 418003

Changsha, P.R. China

Full list of author information is

available at the end of the article

\begin{abstract}
We consider the following Schrödinger-Bopp-Podolsky problem:

$$
\begin{cases}-\Delta u+V(x) u+\phi u=\lambda f(u)+|u|^{4} u, & \text { in } \mathbb{R}^{3}, \\ -\Delta \phi+\Delta^{2} \phi=u^{2}, & \text { in } \mathbb{R}^{3} .\end{cases}
$$

We prove the existence result without any growth and Ambrosetti-Rabinowitz conditions. In the proofs, we apply a cut-off function, the mountain pass theorem, and Moser iteration.
\end{abstract}

MSC: $35 J 50 ; 35 Q 60$

Keywords: Schrödinger-Bopp-Podolsky problem; Mountain pass theorem; Moser iteration

\section{Introduction and statement of results}

In this paper, we deal with the following Schrödinger-Bopp-Podolsky system with critical growth:

$$
\begin{cases}-\Delta u+V(x) u+\phi u=\lambda f(u)+|u|^{4} u, & \text { in } \mathbb{R}^{3}, \\ -\Delta \phi+\Delta^{2} \phi=u^{2}, & \text { in } \mathbb{R}^{3},\end{cases}
$$

where $\lambda>0$ is a parameter. Systems such as (1.1) have been introduced in [1] as a model describing solitary waves for nonlinear stationary equations of Schrödinger type interacting with an electrostatic field in the Bopp-Podolsky electromagnetic theory and are usually known as Schrödinger-Bopp-Podolsky systems. We refer to [2-7] for a more detailed description of the physical aspects of this problem. In this paper, we suppose that $V, f$ satisfy the following assumptions:

$$
\left(V_{1}\right) \quad V \in C\left(\mathbb{R}^{3}, \mathbb{R}\right), V_{0}=\inf _{x \in \mathbb{R}^{3}} V(x)>0 .
$$

(c) The Author(s) 2020. This article is licensed under a Creative Commons Attribution 4.0 International License, which permits use, sharing, adaptation, distribution and reproduction in any medium or format, as long as you give appropriate credit to the original author(s) and the source, provide a link to the Creative Commons licence, and indicate if changes were made. The images or other third party material in this article are included in the article's Creative Commons licence, unless indicated otherwise in a credit line to the material. If material is not included in the article's Creative Commons licence and your intended use is not permitted by statutory regulation or exceeds the permitted use, you will need to obtain permission directly from the copyright holder. To view a copy of this licence, visit http://creativecommons.org/licenses/by/4.0/. 
$\left(V_{2}\right)$ For any $T>0$, there exists $r>0$ such that

$$
\lim _{|y| \rightarrow \infty} \operatorname{meas}\left(\left\{x \in \mathbb{R}^{3}:|x-y| \leq r, V(x) \leq T\right\}\right)=0,
$$

where meas $(A)$ is the Lebesgue measure of $A$.

$\left(f_{1}\right) f \in C(\mathbb{R})$ and $f(u)=o(u)$ as $u \rightarrow 0$.

$\left(f_{2}\right) f(u) / u \rightarrow+\infty$ as $|u| \rightarrow \infty$.

The solution to (1.1) is understood in the weak sense, that is, a pair $(u, \phi) \in H^{1}\left(\mathbb{R}^{3}\right) \times \mathcal{D}$ is a solution to (1.1) if

$$
\begin{aligned}
& \int_{\mathbb{R}^{3}}(\nabla u \nabla v+V(x) u v+\phi u v) d x=\int_{\mathbb{R}^{3}}\left(\lambda f(u) v+|u|^{4} u v\right) d x, \quad \forall v \in H^{1}\left(\mathbb{R}^{3}\right), \\
& \int_{\mathbb{R}^{3}} \nabla \phi \nabla \omega d x+\int_{\mathbb{R}^{3}} \Delta \phi \Delta \omega d x=\int_{\mathbb{R}^{3}} \phi u^{2} d x, \quad \forall \omega \in \mathcal{D},
\end{aligned}
$$

where $\mathcal{D}$ is a function space that will be introduced in Sect. 2 . To the best of our knowledge, there are very few papers related to the existence of solutions to problem (1.1). In [1], d'Avenia and Siciliano studied the following Schrödinger-Bopp-Podolsky equation:

$$
\begin{cases}-\Delta u+\omega u+q^{2} \phi u=|u|^{p-2} u, & \text { in } \mathbb{R}^{3}, \\ -\Delta \phi+a^{2} \Delta^{2} \phi=4 \pi u^{2}, & \text { in } \mathbb{R}^{3} .\end{cases}
$$

The authors give existence and nonexistence results, depending on the parameters $p$ and $q$. Moreover, they also show that in the radial case, the solutions that they find tend to solutions of the classical Schrödinger-Poisson system as $a \rightarrow 0$.

When $a=0,(1.2)$ reduces to the following well-known Schrödinger-Poisson equation

$$
\begin{cases}-\Delta u+\omega u+q^{2} \phi u=|u|^{p-2} u, & \text { in } \mathbb{R}^{3}, \\ -\Delta \phi=4 \pi u^{2}, & \text { in } \mathbb{R}^{3},\end{cases}
$$

that has been extensively studied in the past few decades. There have been many existence and nonexistence results in the past decades. For some recent results, we refer the readers to [8-13] and the references therein. We now summarize our main results as follows.

Theorem 1.1 Suppose that assumptions $\left(f_{1}\right)-\left(f_{2}\right)$ and $\left(V_{1}\right)-\left(V_{2}\right)$ are satisfied. Then there exists $\lambda_{1}>0$ such that, for any $\lambda \in\left(0, \lambda_{1}\right)$, problem (1.1) has a nontrivial solution.

Remark 1.1 We note that the usual growth condition and the Ambrosetti-Rabinowitz condition are not needed in our result. Moreover, $f$ is allowed to be sign-changing.

Remark 1.2 A typical example of a function satisfying assumptions $\left(f_{1}\right)-\left(f_{2}\right)$ is given by $f(t)=|t|^{q-2} t, q>6$. Furthermore, our conclusion holds for general supercritical nonlinearity.

The proof will be carried out by variational methods. Since the Sobolev embedding $H^{1}\left(\mathbb{R}^{3}\right) \hookrightarrow L^{s}\left(\mathbb{R}^{3}\right)$ is not compact, the main difficulty is the lack of compactness. Since we 
do not assume Ambrosetti-Rabinowitz or growth conditions on $f$, we first make a suitable modification on $f$, solve the modified problem, and then check that, for small enough $\lambda$, the solutions of the modified problem are also the solutions of the original problem. We note that even for the modified problem it is not easy to obtain compactness in view of the critical growth of the nonlinearity. To overcome the loss of compactness for the energy functional, we shall verify that the Palais-Smale condition is regained when the energy functional is below a suitable level.

The rest of this paper is organized as follows. In Sect. 2, we state some preliminary notations, modify the original problem, and prove the existence result of the modified problem. In Sect. 3, we prove Theorem 1.1.

\section{Preliminaries and the modified problem}

In this paper, we use the following notation:

- $H^{1}\left(\mathbb{R}^{3}\right)$ is the usual Sobolev space with an inner product and norm given by

$$
\langle u, v\rangle_{H^{1}\left(\mathbb{R}^{3}\right)}:=\int_{\mathbb{R}^{3}}(\nabla u \nabla v+u v) d x, \quad\|\cdot\|_{H^{1}\left(\mathbb{R}^{3}\right)}=\left(\int_{\mathbb{R}^{3}}\left(|\nabla u|^{2}+|u|^{2}\right) d x\right)^{\frac{1}{2}} .
$$

- $L^{p}\left(\mathbb{R}^{3}\right), 1 \leq p \leq+\infty$, denotes a Lebesgue space, and the norm in $L^{p}\left(\mathbb{R}^{3}\right)$ is denoted by $|\cdot|_{p}$

- $D^{1,2}\left(\mathbb{R}^{3}\right)$ is the completion of $C_{0}^{\infty}\left(\mathbb{R}^{3}\right)$ with respect to the norm

$$
\|u\|_{D^{1,2}\left(\mathbb{R}^{3}\right)}^{2}=\int_{\mathbb{R}^{3}}|\nabla u|^{2} d x
$$

- $C, C_{i}$ denote (possible different) any positive constant.

- $H^{-1}$ denotes the dual space of $H^{1}\left(\mathbb{R}^{3}\right)$.

In this section, we summarize some fundamental properties of the operator $-\Delta+\Delta^{2}$ and functional space $\mathcal{D}$. The $\mathcal{D}$ is defined by the completion of $C_{0}^{\infty}\left(\mathbb{R}^{3}\right)$ equipped with the norm $\|\cdot\|_{\mathcal{D}}$ induced by the scalar product

$$
\langle u, v\rangle_{\mathcal{D}}:=\int_{\mathbb{R}^{3}} \nabla u \nabla v d x+\int_{\mathbb{R}^{3}} \Delta u \Delta v d x
$$

For more details, we refer the reader to [1].

It is easy to show that $\mathcal{D}$ is a Hilbert space continuously embedded into $D^{1,2}\left(\mathbb{R}^{3}\right)$ and consequently in $L^{\infty}\left(\mathbb{R}^{3}\right)$, see [1].

Lemma 2.1 ([1, Lemma 3.2]) The space $C_{0}^{\infty}\left(\mathbb{R}^{3}\right)$ is dense in

$$
\mathcal{A}:=\left\{\phi \in D^{1,2}\left(\mathbb{R}^{3}\right): \Delta \phi \in L^{2}\left(\mathbb{R}^{3}\right)\right\}
$$

normed by $\sqrt{\langle\phi, \phi\rangle_{\mathcal{D}}}$ and, therefore, $\mathcal{D}=\mathcal{A}$.

For every fixed $u \in H^{1}\left(\mathbb{R}^{3}\right)$, the Riesz theorem implies that there exists a unique solution $\phi_{u} \in \mathcal{D}$ such that

$$
-\Delta \phi+\Delta^{2} \phi=u^{2} .
$$


In order to write explicitly this solution, we consider

$$
\mathcal{K}(x)=\frac{1-e^{-|x|}}{4 \pi|x|}
$$

and $\mathcal{K}(x-y)$ is the fundamental solution of the equation $-\Delta \phi+\Delta^{2} \phi=\delta_{y}$. See [7, formula 2.6] and [1, Lemma 3.3] for more properties of $\mathcal{K}(x)$. Then, the unique solution in $\mathcal{D}$ to the second equation in (1.1) is

$$
\phi_{u}(x):=\mathcal{K} * u^{2}=\frac{1}{4 \pi} \int_{\mathbb{R}^{3}} \frac{1-e^{-|x-y|}}{|x-y|} u^{2}(y) d y .
$$

The function $\phi_{u}$ possesses the following properties (see [1]).

Lemma 2.2 For every $u \in H^{1}\left(\mathbb{R}^{3}\right)$, we have:

(i) $\phi_{u} \geq 0$ for all $u \in H^{1}\left(\mathbb{R}^{3}\right)$;

(ii) $\left\|\phi_{u}\right\|_{\mathcal{D}} \leq C\|u\|^{2}, \int_{\mathbb{R}^{3}} \phi_{u} u^{2} d x \leq C\|u\|_{12 / 5}^{4}$;

(iii) if $u_{n} \rightarrow u$ in $H^{1}\left(\mathbb{R}^{3}\right)$, then $\phi_{u_{n}} \rightarrow \phi_{u}$ in $\mathcal{D}$;

(iv) for every $y \in \mathbb{R}^{3}, \phi_{u(++y)}=\phi_{u}(\cdot+y)$;

(v) $\phi_{u}$ is the unique minimizer of the functional

$$
E(\phi)=\frac{1}{2}\|\nabla \phi\|_{2}^{2}+\frac{1}{2}\|\Delta \phi\|_{2}^{2}-\int_{\mathbb{R}^{3}} \phi u^{2} d x, \quad \phi \in \mathcal{D} .
$$

Substituting (2.1) into (1.1), we obtain

$$
-\Delta u+V(x) u+\phi_{u} u=\lambda f(u)+|u|^{4} u, \quad u \in H^{1}\left(\mathbb{R}^{3}\right) .
$$

Then we define a smooth functional $\Phi: H^{1}\left(\mathbb{R}^{3}\right) \rightarrow \mathbb{R}$ by setting

$$
\Phi(u)=\int_{\mathbb{R}^{3}} \phi_{u}(x) u^{2}(x) d x .
$$

In fact, functional $\Phi$ possesses the following useful BL-splitting properties, similar to the Brézis-Lieb lemma [14].

Lemma 2.3 Let $u_{n} \rightarrow u$ in $H^{1}\left(\mathbb{R}^{3}\right)$ and $u_{n} \rightarrow$ u a.e. in $\mathbb{R}^{3}$, then

$$
\Phi\left(u_{n}-u\right)=\Phi\left(u_{n}\right)-\Phi(u)+o(1), \quad \text { as } n \rightarrow \infty,
$$

where $\Phi$ is defined by (2.2).

Proof Since $\mathcal{K}(x) \in L^{\tau}\left(\mathbb{R}^{3}\right)$ for $\tau \in(3,+\infty]$, together with $u_{n} \rightarrow u$ in $H^{1}\left(\mathbb{R}^{3}\right)$ and $u_{n} \rightarrow u$ a.e. in $\mathbb{R}^{3}$, we have

$$
\phi_{u_{n}-u}=\phi_{u_{n}}-\phi_{u}+o(1) .
$$

By Lemma $2.2(\mathrm{v})$, we obtain that

$$
\left\langle\phi_{u}, \phi_{u}\right\rangle_{\mathcal{D}}=\Phi(u), \quad \forall u \in H^{1}\left(\mathbb{R}^{3}\right) .
$$


Consequently, by (2.3) and Lemma 2.2 (ii)-(iii), we obtain that

$$
\begin{aligned}
\Phi\left(u_{n}-u\right) & =\left\langle\phi_{u_{n}-u}, \phi_{u_{n}-u}\right\rangle_{\mathcal{D}} \\
& =\left\langle\phi_{u_{n}}-\phi_{u}+o(1), \phi_{u_{n}}-\phi_{u}+o(1)\right\rangle_{\mathcal{D}} \\
& =\left\langle\phi_{u_{n}}, \phi_{u_{n}}\right\rangle_{\mathcal{D}}-2\left\langle\phi_{u_{n}}, \phi_{u}\right\rangle_{\mathcal{D}}+\left\langle\phi_{u}, \phi_{u}\right\rangle_{\mathcal{D}}+o(1) \\
& =\Phi\left(u_{n}\right)-\Phi(u)+o(1) .
\end{aligned}
$$

The proof is complete.

We shall search critical points for the functional

$$
I_{\lambda}(u)=\frac{1}{2} \int_{\mathbb{R}^{3}}\left(|\nabla u|^{2}+V(x) u^{2}\right) d x+\frac{1}{4} \Phi(u)-\int_{\mathbb{R}^{3}}\left(\lambda F(u)+\frac{1}{6}|u|^{6}\right) d x,
$$

where $F(t)=\int_{0}^{t} f(s) d s$, as solutions to (1.1). It is well defined on the Hilbert space

$$
X=\left\{u \in H^{1}\left(\mathbb{R}^{3}\right): \int_{\mathbb{R}^{3}} V(x) u^{2} d x<+\infty\right\}
$$

and has the inner product and norm

$$
\langle u, v\rangle=\int_{\mathbb{R}^{3}}(\nabla u \nabla v+V(x) u v) d x, \quad\|u\|=\langle u, u\rangle^{\frac{1}{2}} .
$$

It is well known under assumptions $\left(V_{1}\right)$ and $\left(V_{2}\right)$ that we have the following compactness lemma see [15] or [16].

Lemma 2.4 Suppose that assumptions $\left(V_{1}\right)$ and $\left(V_{2}\right)$ are satisfied. Then the embedding from $X$ into $L^{s}\left(\mathbb{R}^{3}\right)$ is compact for $s \in[2,6)$.

Since $f$ is continuous, we have $I_{\lambda} \in C^{1}(X, \mathbb{R})$ and

$$
\left\langle I_{\lambda}^{\prime}(u), v\right\rangle=\int_{\mathbb{R}^{3}}\left(\nabla u \nabla v+V(x) u v+\phi_{u} u v\right) d x-\int_{\mathbb{R}^{3}}\left(\lambda f(u) v+|u|^{4} u v\right) d x, \quad \forall u, v \in X .
$$

Since $\left(f_{1}\right)$ and $\left(f_{2}\right)$ imply that $\lim _{t \rightarrow 0^{+}} \frac{f(t)}{t}=0, \lim _{t \rightarrow+\infty} \frac{f(t)}{t}=+\infty$, we can introduce a truncated function. Let $T>0$ be large enough such that $f(T)>0$ according to $\left(f_{2}\right)$. We set

$$
g_{T}(t)= \begin{cases}f(t), & 0 \leq t \leq T \\ C_{T} t^{p-1}, & t>T, \\ 0, & t \leq 0,\end{cases}
$$

where $C_{T}=f(T) / T^{p-1}, p \in(4,6)$. Based on assumptions $\left(f_{1}\right)$ and $\left(f_{2}\right)$ it is easy to show that $g_{T}(t)$ is a continuous function and satisfies the following properties:

$\left(g_{1}\right) \lim _{t \rightarrow 0^{+}} \frac{g_{T}(t)}{t}=0$.

$\left(g_{2}\right) \lim _{t \rightarrow+\infty} \frac{G_{T}(t)}{t^{4}}=+\infty$, where $G(t)=\int_{0}^{t} g(s) d s$.

$\left(g_{3}\right)\left|g_{T}(t)\right| \leq C_{T}^{*}|t|+C_{T}|t|^{p-1}$, where $C_{T}^{*}=\max _{t \in[0, T]}|f(t)| / t$.

$\left(g_{4}\right)$ There exists $\mu=\mu(T)>0$ such that $\operatorname{tg}_{T}(t)-4 G_{T}(t) \geq-\mu t^{2}$ for all $t \geq 0$. 
Now we obtain the modified problem

$$
\left\{\begin{array}{l}
-\Delta u+V(x) u+\phi_{u} u=\lambda g_{T}(u)+|u|^{4} u, \quad x \in \mathbb{R}^{3}, \\
u \in X, \quad u(x)>0 .
\end{array}\right.
$$

We shall search critical points for the functional

$$
I_{\lambda, T}(u)=\frac{1}{2} \int_{\mathbb{R}^{3}}\left(|\nabla u|^{2}+V(x) u^{2}\right) d x+\frac{1}{4} \Phi(u)-\int_{\mathbb{R}^{3}}\left(\lambda G_{T}(u)+\frac{1}{6}|u|^{6}\right) d x,
$$

as solutions to (2.4). Since $g_{T}$ is continuous, we have $I_{\lambda, T} \in C^{1}(X, \mathbb{R})$ and, for any $u, v \in X$,

$$
\left\langle I_{\lambda, T}^{\prime}(u), v\right\rangle=\int_{\mathbb{R}^{3}}\left(\nabla u \nabla v+V(x) u v+\phi_{u} u v\right) d x-\int_{\mathbb{R}^{3}}\left(\lambda g_{T}(u) v+|u|^{4} u v\right) d x
$$

The next lemma shows that the functional $I_{\lambda, T}(u)$ satisfies the mountain pass geometry[14].

Lemma 2.5 The functional $I_{\lambda, T}(u)$ satisfies the following conditions:

(i) there exist $\alpha, \rho>0$ such that $I_{\lambda, T}(u) \geq \alpha$ with $\|u\|=\rho$;

(ii) there exists $e \in X$ such that $\|e\|>\rho$ and $I_{\lambda, T}(e)<0$.

Proof For any $u \in X \backslash\{0\}$ and $\epsilon>0$ small, it follows from $\left(g_{1}\right)$ and $\left(g_{3}\right)$ that

$$
\left|g_{T}(t)\right| \leq \epsilon|t|+C_{\epsilon}|t|^{5}
$$

and

$$
\left|G_{T}(t)\right| \leq \frac{\epsilon}{2}|t|^{2}+\frac{C_{\epsilon}}{6}|t|^{6} .
$$

Thus

$$
\begin{aligned}
I_{\lambda, T}(u) & \geq \frac{1}{2}\|u\|^{2}+\frac{1}{4} \Phi(u)-\int_{\mathbb{R}^{3}}\left(\frac{\lambda \epsilon}{2}|u|^{2}+\frac{1+\lambda C_{\epsilon}}{6}|u|^{6}\right) d x \\
& \geq \frac{1}{2}\|u\|^{2}-C \epsilon\|u\|^{2}-C\|u\|^{6}
\end{aligned}
$$

by Lemma 2.2 (i) and the Sobolev embedding $X \hookrightarrow L^{s}\left(\mathbb{R}^{3}\right)$ for $s \in[2,6]$. Since $\epsilon$ is arbitrarily small, there exist $\rho>0$ and $\alpha>0$ such that $I_{\lambda, T}(u) \geq \alpha>0$ for $\|u\|=\rho$.

Let us check (ii). From $\left(g_{2}\right)$, for any $M>0$, there exists $r_{M}>0$ such that

$$
G_{T}(t) \geq M t^{4}, \quad \forall t \geq r_{M}
$$

Together with $\left(g_{1}\right)$ and $\left(g_{3}\right)$, this implies that, for any $M>0$, there exists a constant $C_{M}>0$ such that

$$
G_{T}(t) \geq M t^{4}-C_{M} t^{2}, \quad \forall t>0 .
$$


Then, for each $u \in X \backslash\{0\}$ and $t>0$, we obtain that

$$
I_{\lambda, T}(t u) \leq \frac{t^{2}}{2}\|u\|^{2}+\frac{t^{4}}{4} \Phi(u)-\lambda M t^{4} \int_{\mathbb{R}^{3}}|u|^{4} d x+\lambda C_{M} t^{2} \int_{\mathbb{R}^{3}}|u|^{2} d x-\frac{t^{6}}{6} \int_{\mathbb{R}^{3}}|u|^{6} d x
$$

The step is proved by taking $e=t_{0} u$ with $t_{0}>0$ large enough.

Now, in view of Lemma 2.5, we can apply a version of the mountain pass theorem without the $(P S)$ condition to obtain a sequence $\left\{u_{n}\right\}$ such that

$$
I_{\lambda, T}\left(u_{n}\right) \rightarrow c_{\lambda, T}, \quad\left\|I_{\lambda, T}^{\prime}\left(u_{n}\right)\right\|_{X^{-1}} \rightarrow 0
$$

As in [14], we define

$$
c_{\lambda, T}=\inf _{\gamma \in \Gamma} \max _{t \in[0,1]} I_{\lambda, T}(\gamma(t))
$$

where

$$
\Gamma=\left\{\gamma \in C\left([0,1], H^{1}\left(\mathbb{R}^{3}\right)\right): \gamma(0)=0, I_{\lambda, T}(\gamma(1))<0\right\} .
$$

Lemma 2.6 Every sequence satisfying (2.7) is bounded in X.

Proof For every $c \in \mathbb{R}$, let $\left\{u_{n}\right\} \subset X$ be a $(P S)_{c}$ sequence satisfying (2.7). Then, by $\left(g_{4}\right)$, we deduce that

$$
\begin{aligned}
c_{\lambda, T} & +o_{n}(1)\left\|u_{n}\right\| \\
& \geq I_{\lambda, T}\left(u_{n}\right)-\frac{1}{4} I_{\lambda, T}^{\prime}\left(u_{n}\right) u_{n} \\
& =\frac{1}{4}\left\|u_{n}\right\|^{2}+\frac{\lambda}{4} \int_{\mathbb{R}^{3}}\left[g_{T}\left(u_{n}\right) u_{n}-4 G_{T}\left(u_{n}\right)\right] d x+\frac{1}{12} \int_{\mathbb{R}^{3}}\left|u_{n}\right|^{6} d x \\
& \geq \frac{1}{4}\left\|u_{n}\right\|^{2}-\frac{\lambda \mu}{4} \int_{\mathbb{R}^{3}}\left|u_{n}^{+}\right|^{2} d x,
\end{aligned}
$$

where $u_{n}^{+}=\max \left\{u_{n}(x), 0\right\}, u_{n}^{-}=\min \left\{u_{n}(x), 0\right\}, u_{n}(x)=u_{n}^{+}+u_{n}^{-}$. We argue by contradiction that $\left\|u_{n}\right\| \rightarrow+\infty$ as $n \rightarrow \infty$. Let $v_{n}=\frac{u_{n}}{\left\|u_{n}\right\|}, n \geq 1$. According to Lemma $2.4, X \hookrightarrow L^{s}\left(\mathbb{R}^{3}\right)$, $s \in[2,6)$ is compact. We may assume that

$$
\begin{aligned}
& v_{n} \rightarrow v, \quad \text { a.e. in } \mathbb{R}^{3}, \\
& v_{n} \rightarrow v, \quad \text { weakly in } X, \\
& v_{n} \rightarrow v, \quad \text { strongly in } L^{s}\left(\mathbb{R}^{3}\right), 2 \leq s<6 .
\end{aligned}
$$

Moreover, we have

$$
\begin{array}{ll}
v_{n}^{+} \rightarrow v^{+}, & \text {a.e. in } \mathbb{R}^{3}, \\
v_{n}^{+} \rightarrow v^{+}, & \text {weakly in } X, \\
v_{n}^{+} \rightarrow v^{+}, & \text {strongly in } L^{s}\left(\mathbb{R}^{3}\right), 2 \leq s<6 .
\end{array}
$$


From (2.8), we have

$$
o_{n}(1) \geq \frac{1}{4}\left(1-\lambda \mu \int_{\mathbb{R}^{3}}\left|v_{n}^{+}\right|^{2} d x\right)=\frac{1}{4}\left(1-\lambda \mu \int_{\mathbb{R}^{3}}\left|v^{+}\right|^{2} d x\right)+o(1)
$$

We conclude that $v^{+} \neq 0$. Then $u_{n}^{+}=v_{n}^{+}\left\|u_{n}\right\| \rightarrow+\infty$. By (2.5) and (2.7), we obtain

$$
\begin{aligned}
& \frac{I_{\lambda, T}^{\prime}\left(u_{n}\right) u_{n}}{\left\|u_{n}\right\|^{4}} \\
& \quad=o_{n}(1)+\frac{\int_{\mathbb{R}^{3}} \phi_{u_{n}} u_{n}^{2} d x}{\left\|u_{n}\right\|^{4}}-\int_{\mathbb{R}^{3}} \frac{\lambda g_{T}\left(u_{n}^{+}\right) u_{n}^{+}}{\left(u_{n}^{+}\right)^{4}}\left(v_{n}^{+}\right)^{4} d x-\left\|u_{n}\right\|^{2} \int_{\mathbb{R}^{3}}\left|v_{n}\right|^{6} d x \\
& \quad \leq o_{n}(1)+\frac{\int_{\mathbb{R}^{3}} \phi_{u_{n}} u_{n}^{2} d x}{\left\|u_{n}\right\|^{4}}-\int_{\mathbb{R}^{3}} \frac{\lambda g_{T}\left(u_{n}^{+}\right) u_{n}^{+}}{\left(u_{n}^{+}\right)^{4}}\left(v_{n}^{+}\right)^{4} d x .
\end{aligned}
$$

Taking the limit and using Lemma 2.2 (ii), $\left(g_{2}\right)$, and (2.9), we obtain $0 \leq-\infty$, yielding a contradiction. Therefore, $\left\{u_{n}\right\}$ is bounded in $X$.

Now, we denote by $S$ the best constant of the Sobolev embedding $H^{1}\left(\mathbb{R}^{3}\right) \hookrightarrow L^{6}\left(\mathbb{R}^{3}\right)$, i.e.,

$$
S=\inf _{u \in D^{1,2}\left(\mathbb{R}^{3}\right)} \frac{\int_{\mathbb{R}^{3}}|\nabla u|^{2} d x}{\left(\int_{\mathbb{R}^{3}}|u|^{6} d x\right)^{1 / 3}}
$$

As we will show in the following result, the modified functional satisfies the local compactness condition.

Lemma $2.7 I_{\lambda, T}$ satisfies the $(P S)_{c}$ condition at any level $c_{\lambda, T} \in\left(0, \frac{1}{3} S^{\frac{3}{2}}\right)$.

Proof Let $\left\{u_{n}\right\}$ be a $(P S)_{c_{\lambda, T}}$ sequence satisfying (2.7). By Lemma 2.6, $\left\{u_{n}\right\}$ is bounded in $X$. Up to a subsequence, we may assume that

$$
\begin{aligned}
& u_{n} \rightarrow u, \quad \text { a.e. in } \mathbb{R}^{3}, \\
& u_{n} \rightarrow u, \quad \text { weakly in } X, \\
& u_{n} \rightarrow u, \quad \text { strongly in } L^{s}\left(\mathbb{R}^{3}\right), 2 \leq s<6 .
\end{aligned}
$$

Since $\phi: L^{12 / 5}\left(\mathbb{R}^{3}\right) \rightarrow \mathcal{D}$ is continuous, from (2.11) we obtain that

$$
\begin{aligned}
& \phi_{u_{n}} \rightarrow \phi_{u} \quad \text { in } \mathcal{D}, \text { as } n \rightarrow \infty, \\
& \int_{\mathbb{R}^{3}} \phi_{u_{n}} u_{n}^{2} d x \rightarrow \int_{\mathbb{R}^{3}} \phi_{u} u^{2} d x, \quad \text { as } n \rightarrow \infty
\end{aligned}
$$

Using (2.11) and [14, Theorem A.1 ], for any $\varphi \in C_{0}^{\infty}\left(\mathbb{R}^{3}\right) \subset X$, we can obtain that

$$
\begin{aligned}
& \int_{\mathbb{R}^{3}}\left(\nabla u_{n} \nabla \varphi+V(x) u_{n} \varphi\right) d x \rightarrow \int_{\mathbb{R}^{3}}(\nabla u \nabla \varphi+V(x) u \varphi) d x, \\
& \int_{\mathbb{R}^{3}} g_{T}\left(u_{n}\right) \varphi d x \rightarrow \int_{\mathbb{R}^{3}} g_{T}(u) \varphi d x \\
& \int_{\mathbb{R}^{3}}\left|u_{n}\right|^{4} u_{n} \varphi d x \rightarrow \int_{\mathbb{R}^{3}}|u|^{4} u \varphi d x .
\end{aligned}
$$


From (2.11)-(2.12), the Hölder inequality, and the Sobolev embedding, we obtain

$$
\begin{aligned}
\int_{\mathbb{R}^{3}}\left(\phi_{u_{n}} u_{n}-\phi_{u} u\right) \varphi d x= & \int_{\mathbb{R}^{3}} \phi_{u_{n}}\left(u_{n}-u\right) \varphi d x+\int_{\mathbb{R}^{3}}\left(\phi_{u_{n}}-\phi_{u}\right) u \varphi d x \\
\leq & C\left\|\phi_{u_{n}}\right\|_{D^{1,2}\left(\mathbb{R}^{3}\right)}\left|u_{n}-u\right|_{12 / 5}|\varphi|_{12 / 5} \\
& +C\left\|\phi_{u_{n}}-\phi_{u}\right\|_{D^{1,2}\left(\mathbb{R}^{3}\right)}|u|_{12 / 5}|\varphi|_{12 / 5} \\
\rightarrow & 0 .
\end{aligned}
$$

By (2.13)-(2.14), the density of $C_{0}^{\infty}\left(\mathbb{R}^{3}\right)$ in $X$, and (2.7), we can conclude that $I_{\lambda, T}^{\prime}\left(u_{n}\right) \rightarrow$ $I_{\lambda, T}^{\prime}(u)=0$. Let $w_{n}=u_{n}-u$, as $n \rightarrow \infty$. It follows from Lemma 2.3 and the Brezis-Lieb lemma that

$$
I_{\lambda, T}\left(w_{n}\right)=I_{\lambda, T}\left(u_{n}\right)-I_{\lambda, T}(u)=c-I_{\lambda, T}(u)+o(1):=d+o(1)
$$

and $I_{\lambda, T}^{\prime}\left(w_{n}\right) \rightarrow 0$ in $X^{-1}$. We recall that the continuous embedding $X \hookrightarrow L^{s}\left(\mathbb{R}^{3}\right)$ is compact for $2 \leq s<6$. Hence, up to a subsequence, $w_{n} \rightarrow 0$ in $L^{s}\left(\mathbb{R}^{3}\right)$, and

$$
\left\|w_{n}\right\|^{2}+\int_{\mathbb{R}^{3}} \phi_{w_{n}} w_{n}^{2} d x=\int_{\mathbb{R}^{3}} \lambda g_{T}\left(w_{n}\right) w_{n} d x+\int_{\mathbb{R}^{3}} w_{n}^{6} d x+o(1)
$$

By Lemma 2.2(ii), we obtain that

$$
\int_{\mathbb{R}^{3}} \phi_{w_{n}} w_{n}^{2} d x \leq C\left|w_{n}\right|_{12 / 5}^{4} \rightarrow 0
$$

Hence, by $\left(g_{3}\right),(2.15)-(2.16)$, we have

$$
\left\|w_{n}\right\|^{2}=\int_{\mathbb{R}^{3}}\left|w_{n}\right|^{6} d x+o(1)
$$

Since $w_{n} \subset X$ is bounded, we may assume that as $n \rightarrow \infty$

$$
\left\|w_{n}\right\|^{2} \rightarrow b \geq 0, \quad \int_{\mathbb{R}^{3}}\left|w_{n}\right|^{6} d x \rightarrow b \geq 0,
$$

up to a subsequence. Suppose by contradiction that $b>0$. By the Sobolev inequality, we have

$$
\left\|w_{n}\right\|^{2} \geq \int_{\mathbb{R}^{3}}\left|\nabla w_{n}\right|^{2} d x \geq S\left|w_{n}\right|_{6}^{2}
$$

and, therefore, $b \geq S^{\frac{3}{2}}$. Thus

$$
d=\lim _{n \rightarrow \infty} I_{\lambda, T}\left(w_{n}\right)=\left(\frac{1}{2}-\frac{1}{6}\right) b \geq \frac{1}{3} S^{\frac{3}{2}}
$$

which contradicts our assumption. Therefore, $b=0$ and the proof is complete.

To obtain the existence result for problem (2.4) by Lemma 2.7, we need to show that the mountain pass value $c_{\lambda, T}<\frac{1}{3} S^{\frac{3}{2}}$. 
Lemma 2.8 For any $\lambda>0, c_{\lambda, T}<\frac{1}{3} S^{\frac{3}{2}}$.

Proof For $\epsilon>0$, consider the function

$$
U_{\epsilon}(x)=\frac{\left(3 \epsilon^{2}\right)^{\frac{1}{4}}}{\left(\epsilon^{2}+|x|^{2}\right)^{\frac{1}{2}}} .
$$

We recall that $U_{\epsilon}(x)$ satisfies

$$
\begin{cases}-\Delta u=|u|^{4} u, & \text { in } \mathbb{R}^{3}, \\ u \in D^{1,2}\left(\mathbb{R}^{3}\right), u(x)>0, & \text { in } \mathbb{R}^{3},\end{cases}
$$

and

$$
\int_{\mathbb{R}^{3}}\left|\nabla U_{\epsilon}\right|^{2} d x=\int_{\mathbb{R}^{3}}\left|U_{\epsilon}\right|^{6} d x=S^{\frac{3}{2}} .
$$

Let $\psi \in C_{0}^{\infty}\left(\mathbb{R}^{3},[0,1]\right)$ be such that $\psi(x)=1$ for $|x| \leq r$ and $\psi(x)=0$ for $|x| \geq 2 r$. Set $u_{\epsilon}(x)=$ $\psi(x) U_{\epsilon}(x)$. Then, the following asymptotic estimates hold if $\epsilon$ is small enough (see [14]):

$$
\begin{aligned}
& \int_{\mathbb{R}^{3}}\left|\nabla u_{\epsilon}\right|^{2} d x=S^{\frac{3}{2}}+O(\epsilon), \\
& \int_{\mathbb{R}^{3}}\left|u_{\epsilon}\right|^{6} d x=S^{\frac{3}{2}}+O\left(\epsilon^{3}\right), \\
& \int_{\mathbb{R}^{3}}\left|u_{\epsilon}\right|^{s} d x= \begin{cases}O\left(\epsilon^{\frac{s}{2}}\right), & s \in[2,3), \\
O\left(\epsilon^{\frac{s}{2}}|\ln \epsilon|\right), & s=3, \\
O\left(\epsilon^{\frac{6-s}{2}}\right), & s \in(3,6) .\end{cases}
\end{aligned}
$$

Since $I_{\lambda, T}\left(t u_{\epsilon}\right) \rightarrow-\infty$, as $t \rightarrow \infty$, there exists $t_{\epsilon}>0$ such that

$$
I_{\lambda, T}\left(t_{\epsilon} u_{\epsilon}\right)=\sup _{t \geq 0} I_{\lambda, T}\left(t u_{\epsilon}\right)>0 .
$$

We claim that $\left\{t_{\epsilon}\right\}_{\epsilon>0}$ is bounded from below by a positive constant. Otherwise, there exists a sequence $\left\{\epsilon_{n}\right\} \subset \mathbb{R}^{+}$such that $\lim _{n \rightarrow \infty} t_{\epsilon_{n}}=0$ and

$$
I_{\lambda, T}\left(t_{\epsilon_{n}} u_{\epsilon_{n}}\right)=\sup _{t \geq 0} I_{\lambda, T}\left(t u_{\epsilon}\right)
$$

Therefore, $0<\alpha \leq c \leq \lim _{n \rightarrow \infty} I_{\lambda, T}\left(t_{\epsilon_{n}} u_{\epsilon_{n}}\right)=0$, yielding a contradiction. Thus there exists $t_{0}>0$ such that $t_{\epsilon} \geq t_{0}>0$. Moreover, we make the following assertion: $\left\{t_{\epsilon}\right\}_{\epsilon>0}$ is bounded from above. In fact, suppose by contradiction that there exists a subsequence $\left\{t_{\epsilon_{n}}\right\}$ with $t_{\epsilon_{n}} \rightarrow+\infty$. Then, from (2.17)-(2.19), we obtain

$$
0<c_{\lambda, T} \leq I_{\lambda, T}\left(t_{\epsilon_{n}} u_{\epsilon_{n}}\right) \leq C_{1} t_{\epsilon_{n}}^{2}+C_{2} t_{\epsilon_{n}}^{4}-C_{3} t_{\epsilon_{n}}^{6}
$$

Letting $n \rightarrow \infty$ in (2.20), we obtain $0<-\infty$, which is a contradiction. Therefore, $\left\{t_{\epsilon}\right\}_{\epsilon>0}$ is bounded from above. Let

$$
h(t)=\frac{t^{2}}{2} \int_{\mathbb{R}^{3}}\left|\nabla u_{\epsilon}\right|^{2} d x-\frac{t^{6}}{6} \int_{\mathbb{R}^{3}}\left|u_{\epsilon}\right|^{6} d x .
$$


It is easy to see that

$$
\sup _{t \geq 0} h(t)=\frac{1}{3} S^{\frac{3}{2}}+O(\epsilon) .
$$

By $\left(V_{2}\right)$, for $|x| \leq r$, there exists $\beta>0$ such that

$$
|V(x)| \leq \beta
$$

From (2.6), (2.21)-(2.22), and (2.19), we obtain

$$
\begin{aligned}
c_{\lambda, T} \leq & I_{\lambda, T}\left(t_{\epsilon} u_{\epsilon}\right) \\
\leq & \sup _{t \geq 0} h(t)+\frac{t_{\epsilon}^{2}}{2} \beta \int_{\mathbb{R}^{3}}\left|u_{\epsilon}\right|^{2} d x+\frac{t_{\epsilon}^{4}}{4} \int_{\mathbb{R}^{3}} \phi_{u_{\epsilon}} u_{\epsilon}^{2} d x \\
& -\lambda M t_{\epsilon}^{4} \int_{\mathbb{R}^{3}}\left|u_{\epsilon}\right|^{4} d x+t_{\epsilon}^{2} \lambda C_{M} \int_{\mathbb{R}^{3}}\left|u_{\epsilon}\right|^{2} d x \\
\leq & \sup _{t \geq 0} h(t)+C\left|u_{\epsilon}\right|_{2}^{2}+C\left|u_{\epsilon}\right|_{12 / 5}^{4}-\lambda M C\left|u_{\epsilon}\right|_{4}^{4} \\
\leq & \frac{1}{3} S^{\frac{3}{2}}+C O(\epsilon)+C O\left(\epsilon^{2}\right)-\lambda M C O(\epsilon) .
\end{aligned}
$$

Choosing large enough $M>0$, the conclusion follows from (2.23) for small enough $\epsilon>0$.

Theorem 2.9 For any $\lambda>0, T>0$, problem (2.4) has a nontrivial solution $u_{\lambda}$ with $I_{\lambda, T}\left(u_{\lambda}\right)=c_{\lambda, T}$.

Proof Since the functional $I_{\lambda, T}$ contains the mountain pass geometry and satisfies the $(P S)_{c}$ condition, the mountain pass theorem [14] implies that there exists a critical point $u_{\lambda} \in X$. Moreover, $I_{\lambda, T}\left(u_{\lambda}\right)=c_{\lambda, T} \geq \alpha>0=I(0)$, so that $u_{\lambda}$ is a nontrivial solution.

\section{Proof of Theorem 1.1}

In this section, we prove our main result. Our approach is based on showing that the solution obtained in Theorem 2.9 satisfies the estimate $\left|u_{\lambda}\right|_{\infty} \leq T$. This implies that $u_{\lambda}$ is indeed the solution to the original problem (1.1). The following lemma plays a fundamental role in the study of the existence of the nontrivial solution to problem (1.1), and its proof involves some arguments explored in $[17,18]$ and involves the use of the Nash-Moser method [19].

Lemma 3.1 If $u$ is a critical point of $I_{\lambda, T}$, then $u \in L^{\infty}\left(\mathbb{R}^{3}\right)$ and

$$
|u|_{\infty} \leq C_{0}^{\frac{1}{2(\eta-1)}} \eta^{\eta /(\eta-1)^{2}}\left[\left(\lambda C_{T}^{*}+\alpha(\epsilon, u)\right)\left(1+|u|_{2}\right)^{2}+\lambda C_{T}|u|_{6}^{p-2}\right]^{\frac{1}{2(\eta-1)}}|u|_{6}^{\kappa},
$$

where $C_{0}>0$ and $\kappa \leq 1$ are constants independent of $\lambda$ and $T, \eta=(8-p) / 2$.

Proof Let $A_{k}=\left\{x \in \mathbb{R}^{3},|u|^{s-1} \leq k\right\}, B_{k}=\mathbb{R}^{3} \backslash A_{k}$, where $s>1, k>0$. Define

$$
u_{k}= \begin{cases}u|u|^{2(s-1)}, & x \in A_{k}, \\ k^{2} u, & x \in B_{k}\end{cases}
$$


and

$$
w_{k}= \begin{cases}u|u|^{s-1}, & x \in A_{k} \\ k u, & x \in B_{k}\end{cases}
$$

Then $u_{k}, w_{k} \in X,\left|u_{k}\right| \leq|u|^{2 s-1}$, and $w_{k}^{2}=u u_{k} \leq|u|^{2 s}$. It is easy to check that

$$
\begin{aligned}
& \nabla u_{k}= \begin{cases}(2 s-1)|u|^{2 s-2} \nabla u, & x \in A_{k}, \\
k^{2} \nabla u, & x \in B_{k},\end{cases} \\
& \nabla w_{k}= \begin{cases}s|u|^{s-1} \nabla u, & x \in A_{k}, \\
k \nabla u, & x \in B_{k},\end{cases}
\end{aligned}
$$

and

$$
\int_{\mathbb{R}^{3}}\left(\left|\nabla w_{k}\right|^{2}-\nabla u \nabla u_{k}\right) d x=(s-1)^{2} \int_{A_{k}}|u|^{2(s-1)}|\nabla u|^{2} d x
$$

Observe that

$$
\begin{aligned}
& \int_{\mathbb{R}^{3}} \nabla u \cdot \nabla u_{k} d x \\
& \quad(2 s-1) \int_{A_{k}}|u|^{2(s-1)}|\nabla u|^{2} d x+k^{2} \int_{B_{k}}|\nabla u|^{2} d x \\
& \quad \geq(2 s-1) \int_{A_{k}}|u|^{2(s-1)}|\nabla u|^{2} d x
\end{aligned}
$$

Therefore, by (3.1)-(3.2), we obtain that $\int_{\mathbb{R}^{3}} \nabla u \cdot \nabla u_{k} d x \geq 0$ and

$$
\int_{\mathbb{R}^{3}}\left|\nabla w_{k}\right|^{2} d x \leq s^{2} \int_{\mathbb{R}^{3}} \nabla u \cdot \nabla u_{k} d x
$$

Using $u_{k}$ as a test function in (2.5), we obtain

$$
\int_{\mathbb{R}^{3}}\left(\nabla u \nabla u_{k}+V(x) u u_{k}+\phi_{u} u u_{k}\right) d x=\int_{\mathbb{R}^{3}}\left(\lambda g_{T}(u) u_{k}+|u|^{4} u u_{k}\right) d x
$$

Together with (3.3), this shows that

$$
\int_{\mathbb{R}^{3}}\left|\nabla w_{k}\right|^{2} d x \leq s^{2}\left(\int_{\mathbb{R}^{3}} \lambda g_{T}(u) u_{k} d x+\int_{\mathbb{R}^{3}}|u|^{4} u u_{k} d x\right) .
$$

By a version of the Brézis-Kato lemma, as in [20, Lemma 2.5], for any $\epsilon>0$, there exists $\alpha(\epsilon, u)$ such that

$$
\int_{\mathbb{R}^{3}}|u|^{4} w_{k}^{2} d x \leq \epsilon \int_{\mathbb{R}^{3}}\left|\nabla w_{k}\right|^{2} d x+\alpha(\epsilon, u) \int_{\mathbb{R}^{3}}\left|w_{k}\right|^{2} d x
$$


Choosing $\epsilon=\frac{1}{2 s^{2}}$, we obtain

$$
\int_{\mathbb{R}^{3}}\left|\nabla w_{k}\right|^{2} d x \leq 2 s^{2}\left(\int_{\mathbb{R}^{3}} \lambda g_{T}(u) u_{k} d x+\alpha(\epsilon, u) \int_{\mathbb{R}^{3}}\left|w_{k}\right|^{2} d x\right) .
$$

By $\left(g_{3}\right)$ and $w_{k}^{2}=u u_{k}$, we obtain

$$
\left|g_{T}(u) u_{k}\right| \leq C_{T}^{*} w_{k}^{2}+C_{T}|u|^{p-2} w_{k}^{2}
$$

By the Sobolev embedding theorem, (3.4)-(3.5), and the Hölder inequality, we obtain

$$
\begin{aligned}
\left(\int_{A_{k}}\left|w_{k}\right|^{6}\right)^{1 / 3} & \leq S^{-1} \int_{\mathbb{R}^{3}}\left|\nabla w_{k}\right|^{2} d x \\
& \leq S^{-1} 2 s^{2}\left[\int_{\mathbb{R}^{3}} \lambda\left(C_{T}^{*} w_{k}^{2}+C_{T}|u|^{p-2} w_{k}^{2}\right) d x+\alpha(\epsilon, u) \int_{\mathbb{R}^{3}}\left|w_{k}\right|^{2} d x\right] \\
& \leq S^{-1} 2 s^{2}\left[\left(\lambda C_{T}^{*}+\alpha(\epsilon, u)\right)\left|w_{k}\right|_{2}^{2}+\lambda C_{T}|u|_{6}^{p-2}\left|w_{k}\right|_{2 q}^{2}\right]
\end{aligned}
$$

where $q=\frac{6}{8-p} \in\left(\frac{3}{2}, 3\right)$. Recalling that $\left|w_{k}\right| \leq|u|^{s}$ and $\left|w_{k}\right|=|u|^{s}$ for $x \in A_{k}$, together with (3.6), we obtain that

$$
\left(\int_{A_{k}}|u|^{6 s}\right)^{1 / 3} \leq S^{-1} 2 s^{2}\left[\left(\lambda C_{T}^{*}+\alpha(\epsilon, u)\right)|u|_{2 s}^{2 s}+\lambda C_{T}|u|_{6}^{p-2}|u|_{2 s q}^{2 s}\right]
$$

Moreover, by the interpolation inequality, we obtain $|u|_{2 s} \leq|u|_{2}^{1-\sigma}|u|_{2 q s}^{\sigma}$, where $\sigma \in(0,1)$ satisfying $\frac{1}{2 s}=\frac{1-\sigma}{2}+\frac{\sigma}{2 s q}$, that is, $\sigma=\frac{q(s-1)}{q s-1}$. Consequently, since $2 s(1-\sigma)=2+\frac{2(1-s)}{q s-1}<2$, we obtain

$$
|u|_{2 s}^{2 s} \leq|u|_{2}^{2 s(1-\sigma)}|u|_{2 s q}^{2 s \sigma} \leq\left(1+|u|_{2}\right)^{2}|u|_{2 s q}^{2 s \sigma} .
$$

Letting $k \rightarrow \infty$, from (3.7)-(3.8), we obtain

$$
\begin{aligned}
|u|_{6 s} & \leq\left(S^{-1} 2 s^{2}\right)^{\frac{1}{2 s}}\left[\left(\lambda C_{T}^{*}+\alpha(\epsilon, u)\right)\left(1+|u|_{2}\right)^{2}|u|_{2 s q}^{2 s \sigma}+\lambda C_{T}|u|_{6}^{p-2}|u|_{2 s q}^{2 s}\right]^{\frac{1}{2 s}} \\
& \leq C_{0}^{\frac{1}{2 s}} s^{\frac{1}{s}}\left[\left(\lambda C_{T}^{*}+\alpha(\epsilon, u)\right)\left(1+|u|_{2}\right)^{2}+\lambda C_{T}|u|_{6}^{p-2}\right]^{\frac{1}{2 s}}|u|_{2 s q}^{\kappa}
\end{aligned}
$$

where $\kappa=\{\sigma, 1\}, C_{0}=\max \left\{2 S^{-1}, 1\right\}$. Let $\eta=\frac{6}{2 q}$, then $\eta \in(1,2)$. We now perform $j$ iterations by setting $s_{j}=\eta^{j}$ in (3.9) and obtain that

$$
\begin{aligned}
& |u|_{6 \eta^{j}} \\
& \quad \leq C_{0}^{\frac{1}{2} \sum_{j=1}^{\infty} \frac{1}{\eta^{j}}} \eta^{\sum_{j=1}^{\infty} \frac{j}{\eta^{j}}}\left[\left(\lambda C_{T}^{*}+\alpha\right)\left(1+|u|_{2}\right)^{2}+\lambda C_{T}|u|_{6}^{p-2}\right]^{\frac{1}{2} \sum_{j=1}^{\infty} \frac{1}{\eta^{j}}|u|_{6}^{\kappa_{1} \cdots \kappa_{j}}}
\end{aligned}
$$

where $\sigma_{j}=q\left(\eta_{j}-1\right) /\left(q \eta^{j}-1\right)<1, \kappa_{j}=\left\{\sigma_{j}, 1\right\} \leq 1$. By a simple calculation, we obtain that $\sum_{j=1}^{\infty} 1 / \eta^{j}=\frac{1}{\eta-1}, \sum_{j=1}^{\infty} j / \eta^{j}=\frac{\eta}{(\eta-1)^{2}}$. We will divide the study of $|u|_{\infty}$ into two cases.

(i) If $|u|_{6} \geq 1$, then $|u|_{6}^{\kappa_{1} \kappa_{2} \cdots \kappa_{j}} \leq|u|_{6}$. Letting $j \rightarrow \infty$ in (3.10), we obtain

$$
|u|_{\infty} \leq C_{0}^{\frac{1}{2(\eta-1)}} \eta^{\frac{\eta}{(\eta-1)^{2}}}\left[\left(\lambda C_{T}^{*}+\alpha(\epsilon, u)\right)\left(1+|u|_{2}\right)^{2}+\lambda C_{T}|u|_{6}^{p-2}\right]^{\frac{1}{2(\eta-1)}}|u|_{6}
$$


(ii) If $|u|_{6}<1$ from $\sigma_{j}=\frac{q\left(\eta^{j}-1\right)}{q \eta^{j}-1} \geq 1-\frac{1}{\eta^{j}}$ and $\kappa_{j}=\left\{\sigma_{j}, 1\right\}$, then for any $j \in \mathbb{N}$, we obtain

$$
0<\sigma_{1} \sigma_{2} \cdots \sigma_{j} \leq \kappa_{1} \kappa_{2} \cdots \kappa_{j}
$$

It can be easily seen that $\ln (1-s) \geq-s-\frac{s^{2}}{2(1-s)^{2}}, s \in(0,1)$, implying that

$$
\sum_{i=1}^{j} \ln \kappa_{i} \geq \sum_{i=1}^{j} \ln \sigma_{i} \geq-\sum_{i=1}^{j} \frac{1}{\eta^{i}}-\frac{1}{2} \sum_{i=1}^{j} \frac{1}{\left(\eta^{i}-1\right)^{2}} .
$$

By a direct calculation, we can conclude that

$$
\sum_{i=1}^{\infty} \frac{1}{\left(\eta^{i}-1\right)^{2}} \leq \frac{\eta^{2}}{\left(\eta^{2}-1\right)(\eta-1)^{2}}
$$

Hence, we obtain that

$$
\sum_{i=1}^{\infty} \ln \kappa_{i} \geq-\frac{1}{\eta-1}-\frac{\eta^{2}}{2\left(\eta^{2}-1\right)(\eta-1)^{2}}:=\theta
$$

Therefore, $\kappa_{1} \kappa_{2} \cdots \kappa_{j} \geq e^{\theta}, \forall j \in \mathbb{N}$. Consequently, by $|u|_{6}<1$ we obtain that $|u|_{6}^{\kappa_{1} \kappa_{2} \cdots \kappa_{j}} \leq$ $|u|_{6}^{e^{\theta}}$. Similarly, letting $j \rightarrow \infty$ in (3.10), we obtain

$$
|u|_{\infty} \leq C_{0}^{\frac{1}{2(\eta-1)}} \eta^{\frac{\eta}{(\eta-1)^{2}}}\left[\left(\lambda C_{T}^{*}+\alpha(\epsilon, u)\right)\left(1+|u|_{2}\right)^{2}+\lambda C_{T}|u|_{6}^{p-2}\right]^{\frac{1}{2(\eta-1)}}|u|_{6}^{\theta}
$$

Let $\kappa=1$ or $\kappa=e^{\theta} \leq 1$. The proof is complete.

We are now ready to prove the main result of the paper.

Proof of Theorem 1.1 Let $u \in C_{0}^{\infty}\left(\mathbb{R}^{3}\right)$ and $u(x) \leq 0$, then $G_{T}(u)=0$. Hence,

$$
I_{\lambda, T}(t u)=\frac{t^{2}}{2} \int_{\mathbb{R}^{3}}\left(|\nabla u|^{2}+V(x) u^{2}\right) d x+\frac{t^{4}}{4} \Phi(u)-\frac{t^{6}}{6} \int_{\mathbb{R}^{3}}|u|^{6} d x \rightarrow-\infty, \quad \text { as } t \rightarrow+\infty .
$$

Therefore, there exists $t_{0}>0$ such that $I_{\lambda, T}\left(t_{0} u\right)<0$. Let $\gamma(\cdot)=t t_{0} u, t \in[0,1]$, we get $\gamma(t) \in$ $\Gamma$. Since $G_{T}(u)=0$, for all $t \in[0,1]$, we obtain

$$
c_{\lambda, T} \leq \max _{t \in[0,1]} I_{\lambda, T}(\gamma(t)) \leq \max _{t \geq 0}\left(\frac{t^{2}}{2}\|u\|^{2}+\frac{t^{4}}{4} \Phi(u)-\frac{t^{6}}{6}|u|_{6}^{6}\right):=D>0,
$$

where $D$ is a constant independent of $\lambda$ and $T$. From Theorem 2.9, $\left(g_{4}\right)$, and $(V)$, we obtain

$$
4 D \geq 4 c_{\lambda, T}=4 I_{\lambda, T}-\left\langle I_{\lambda, T}^{\prime}\left(u_{\lambda}\right), u_{\lambda}\right\rangle \geq \frac{1}{2}\left\|u_{\lambda}\right\|^{2}+\left(\frac{V_{0}}{2}-\lambda \mu\right)\left|u_{\lambda}\right|_{2}^{2}
$$

We can choose $\lambda_{0}>0$ such that $\frac{V_{0}}{2}-\lambda_{0} \mu>0$. Therefore, based on (3.11), $\left\|u_{\lambda}\right\| \leq 8 D$. Hence, we conclude that $\left|u_{\lambda}\right|_{2} \leq C_{4},\left|u_{\lambda}\right|_{6}^{2} \leq C_{5}$, where $C_{4}, C_{5}>0$ independent of $\lambda, T$. From Lemma 3.1, we obtain

$$
\left|u_{\lambda}\right|_{\infty} \leq C_{0}^{\frac{1}{2(\eta-1)}} \eta^{\frac{\eta}{(\eta-1)^{2}}}\left[\left(\lambda C_{T}^{*}+\alpha(\epsilon, u)\right)\left(1+C_{4}\right)^{2}+\lambda C_{T} C_{5}^{p-2}\right]^{\frac{1}{2(\eta-1)}} C_{5}^{\kappa} .
$$


Hence, we first choose $T>0$ large enough such that

$$
C_{0}^{\frac{1}{2(\eta-1)}} \eta^{\frac{\eta}{(\eta-1)^{2}}}\left[(\alpha(\epsilon, u))\left(1+C_{4}\right)^{2}\right]^{\frac{1}{2(\eta-1)}} C_{5}^{\kappa} \leq \frac{T}{2} .
$$

Since $C_{T}^{*}, C_{T}$ are fixed constants for above $T$, we can choose $\lambda_{1}<\lambda_{0}$ such that

$$
\left|u_{\lambda}\right|_{\infty} \leq C_{0}^{\frac{1}{2(\eta-1)}} \eta^{\frac{\eta}{(\eta-1)^{2}}}\left[\left(\lambda_{1} C_{T}^{*}+\alpha(\epsilon, u)\right)\left(1+C_{4}\right)^{2}+\lambda_{1} C_{T} C_{5}^{p-2}\right]^{\frac{1}{2(\eta-1)}} C_{5}^{\kappa} \leq T .
$$

Then, for $\lambda \in\left(0, \lambda_{1}\right)$, we can obtain $\left|u_{\lambda}\right|_{\infty} \leq T$, and $u_{\lambda}$ is also a solution to the original problem (1.1). The proof of the theorem is now complete.

\section{Acknowledgements}

The authors would like to thank the referees for valuable comments and suggestions for this manuscript.

\section{Funding}

H.B. Chen is supported by the National Natural Science Foundation of China (No. 11671403); J. Yang is supported by the Research Foundation of Education Bureau of Hunan Province, China (No.19B450) and the National Natural Science Foundation of Hunan Province, China (No.2019JJ50473).

\section{Availability of data and materials}

We declare that materials described in the manuscript are freely available to any scientist wishing to use them for noncommercial purposes, without breaching participant confidentiality.

\section{Competing interests}

The authors declare that they have no competing interests.

\section{Authors' contributions}

The authors declare that the study was realized in collaboration with the same responsibility. All authors read and approved the final manuscript.

\section{Author details}

'School of Mathematics and Statistics, Central South University, South Lushan Road, 418003 Changsha, P.R. China.

${ }^{2}$ School of Mathematics and Computational Science, Huaihua University, Huaidong Road, 418008 Huaihua, P.R. China.

\section{Publisher's Note}

Springer Nature remains neutral with regard to jurisdictional claims in published maps and institutional affiliations.

Received: 22 October 2019 Accepted: 17 August 2020 Published online: 28 August 2020

\section{References}

1. d'Avenia, P., Siciliano, G.: Nonlinear Schrödinger equation in the Bopp-Podolsky electrodynamics: solutions in the electrostatic case. J. Differ. Equ. 267(2), 1025-1065 (2019)

2. Born, M.: Modified field equations with a finite radius of the electron. Nature 132, 282 (1933)

3. Born, M.: On the quantum theory of the electromagnetic field. Proc. R. Soc. Lond. Ser. A 143, 410-437 (1934)

4. Born, M., Infeld, L.: Foundations of the new field theory. Nature 132, 1004 (1933)

5. Born, M., Infeld, L.: Foundations of the new field theory. Proc. R. Soc. Lond., Ser. A, Math. Phys. Eng. Sci. 144, $425-451$ (1934)

6. Bopp, F.: Eine Lineare Theorie des Elektrons. Ann. Phys. 430, 345-384 (1940)

7. Podolsky, B.: A generalized electrodynamics. Phys. Rev. 62,68-71 (1942)

8. He, Y., Li, G.: Standing waves for a class of Schrödinger-Poisson equations in $\mathbb{R}^{3}$ involving critical Sobolev exponents. Ann. Acad. Sci. Fenn., Math. 40(2), 729-766 (2015)

9. He, X., Zou, W.M.: Existence and concentration of ground states for Schrödinger-Poisson equations with critical growth. J. Math. Phys. 53(2), 023702 (2012)

10. Chen, Z., Tang, X.H., Zhang, N.: Standing waves for Schrodinger-Poisson system with general nonlinearity. Discrete Contin. Dyn. Syst., Ser. A 39(10), 6103-6129 (2019)

11. Chen, S.T., Tang, X.H., Zhang, N.: Semiclassical states for singularly perturbed Schrodinger-Poisson systems with a general Berestycki-Lions or critical nonlinearity. Topol. Methods Nonlinear Anal. 54(2), 665-683 (2019)

12. Liu, H.L., Chen, H.B., Wang, G.W.: Multiplicity for a 4-sublinear Schrodinger-Poisson system with sign-changing potential via Morse theory. C. R. Math. 354(1), 75-80 (2016)

13. Xie, W.H., Chen, H.B., Shi, H.X.: Multiplicity of positive solutions for Schrodinger-Poisson systems with a critical nonlinearity in $\mathbb{R}^{3}$. Bull. Malays. Math. Sci. Soc. 42(5), 2657-2680 (2019)

14. Williem, M.: Minimax Theorems. Birkhäuser, Boston (1996)

15. Bartsch, T., Wang, Z.Q.: Existence and multiplicity results for some superlinear elliptic problems on $\mathbb{R}^{N}$. Commun. Partial Differ. Equ. 20(9-10), 1725-1741 (1995) 
16. Zou, W.M., Schechter, M.: Critical Point Theory and Its Applications. Springer, New York (2006)

17. Li, G.: Some properties of weak solutions of nonlinear scalar fields equation. Ann. Acad. Sci. Fenn., Math. 14, 27-36 (1989)

18. Li, Y., Geng, Q.: The existence of nontrivial solution to a class of nonlinear Kirchhoff equations without any growth and Ambrosetti-Rabinowitz conditions. Appl. Math. Lett. 96, 153-158 (2019)

19. Gilbarg, D., Trudinger, N.S.: Elliptic Partial Differential Equations of Second Order. Springer, Heidelberg (1983)

20. Zhang, J., Costa, D.G., do'O, J.M.: Semiclassical states of p-Laplacian equations with a general nonlinearity in critical case. J. Math. Phys. 57(7), 071504 (2016)

Submit your manuscript to a SpringerOpen ${ }^{\circ}$ journal and benefit from:

- Convenient online submission

- Rigorous peer review

- Open access: articles freely available online

- High visibility within the field

- Retaining the copyright to your article

Submit your next manuscript at $\gg$ springeropen.com 